\title{
A COMPARISON OF NEBULAR DISTANCE SCALES
}

\author{
M. SAMLAND and J. KÖPPEN \\ Institut für Theoretische Physik und Sternwarte, D-2300 Kiel, Germany \\ A. ACKER \\ Observatoire de Strasbourg, 11, rue de l'Université, 67000 Strasbourg, France \\ and

\section{B. STENHOLM} \\ Lund Observatory, Box 43, S-221 00 Lund, Sweden
}

Determination of the positions of central stars of planetary nebulae in the HRdiagram requires the knowledge of nebular distances. For almost all nebulae, these can only be given in terms of statistical scales. These scales have in common that they assume all nebulae to have the same structure (e.g. constant density) and that a unique ionized mass-radius relation exists. If the mass-radius relation is given by $M_{i o n}=M_{0} \cdot\left(R / R_{0}\right)^{\eta}$, the distance $d(\mathrm{pc})$ of planetary nebulae can be expressed as a function the de-reddened $\mathrm{H} \beta$-flux $\left(\mathrm{erg} \mathrm{cm}^{-2} \mathrm{~s}^{-1}\right)$ and the angular radius $\Theta(\operatorname{arcsec})$ :

$$
\mathrm{d}=\left(\frac{5.5891 \cdot 10^{-6}}{\epsilon \mathrm{F}_{0}(\mathrm{H} \beta)}\right)^{\frac{1}{5-2 \eta}}\left(\frac{\mathrm{M}_{0}}{\mathrm{R}_{0}^{\eta}}\right)^{\frac{2}{5-2 \eta}}\left(\frac{\Theta}{206265}\right)^{\frac{2 \eta-3}{5-2 \eta}}
$$

$M_{0}$ and $R_{0}$ are in solar masses and pc $\left(T_{e}=10000 \mathrm{~K}, \mathrm{He} / \mathrm{H}=0.1\right)$. The parameter $\eta$ characterizes the distance scale: e.g. Shklovsky (1956) $\eta=0$, Maciel \& Pottasch (1980) $\eta=1$, Pottasch (1984) $\eta=3 / 2$, Daub (1982) $\eta=5 / 3$, and Kwok (1985) $\eta=9 / 4$.

For 284 planetary nebulae we take de-reddened $\mathrm{H} \beta$-fluxes from the ESO-Strasbourg spectrophotometric survey (Acker et al. 1989) and the angular diameters from Schneider et al. (1983) and compute the distances with the different methods. Using Ambartzumyan temperatures, we determine the positions of the central stars in the HR-diagram. We find that, for $\eta>2$, the distances show a very large scatter, but for all distance scales with $\eta<2$, the positions agree well with theoretical evolutionary tracks for post-AGB-stars (Schönberner 1983). We note that with $\eta=3 / 2$ the distance of a nebula is only a function of the $\mathrm{H} \beta$-flux which depends on the temperature of the central star. Therefore all central stars are arranged on a crescent-shaped line in the HR-diagram.

\section{References}

Acker A., Köppen K., Stenholm B., Jasniewicz. 1989 A\&AS 80, 201

Daub C. T. 1982, ApJ 260, 612

Kwok S. 1985, ApJ 290, 568

Maciel W. J., Pottasch S. R. 1980, A\&A 88, 1

Pottasch S. R. 1984, Planetary Nebulae, Reidel, Dordrecht

Schneider S. E., Terzian Y., Purgathofer A., Perinotto M., 1983, ApJS 52, 399

Schönberner D.: 1983, ApJ 272, 708

Shklovsky I. S.: 1956, Sov. Astron. J. 33, 108 